\title{
Perfil dos participantes em atividades de extensão universitária associadas às boas práticas agropecuárias na produção de leite caprino
}

Prolife of participants in university extension activities associated with good agricultural practices

in goat milk production

Perfil de partcipantes en actividades de extensíon universitária associadas a buenas prácticas agrícolas en la producción de leche de cabra

Tatiana Labre da Silva

ORCID: https://orcid.org/0000-0003-2011-5000 Universidade Federal Rural do Rio de Janeiro, Brasil

E-mail: labrerural@gmail.com

Daiane de Moura Ribeiro

ORCID: https://orcid.org/0000-0002-2355-0959 Universidade Federal Rural do Rio de Janeiro, Brasil E-mail: moura_daiane@hotmail.com

Bruna dos Santos Lourenço Rodrigues

ORCID: https://orcid.org/0000-0003-2259-1684 Universidade Federal Rural do Rio de Janeiro, Brasil E-mail: brunaslourenco10@gmail.com

Sara Helena Oliveira de Andrade

ORCID: https://orcid.org/0000-0002-7435-2506 Universidade Federal Rural do Rio de Janeiro, Brasil E-mail: sarahelena67@hotmail.com

Júlia dos Santos Fonseca

ORCID: https://orcid.org/0000-0002-2067-2263 Universidade Federal de Viçosa, Brasil

E-mail: julia.agropecuaria@yahoo.com.br

Larissa Santos

ORCID: https://orcid.org/0000-0001-6061-6294 Universidade Federal Rural do Rio de Janeiro, Brasil E-mail: santos.larissa96@gmail.com

Davi de Souza Leal

ORCID: https://orcid.org/0000-0001-9547-3633 Universidade Federal Rural do Rio de Janeiro, Brasil E-mail: davilea19191@gmail.com

Alice Andrade Nóbrega

ORCID: https://orcid.org/0000-0001-2009-8319 Universidade Federal Rural do Rio de Janeiro, Brasil E-mail: andrade815@gmail.com

Willian Bonne Monteiro dos Santos

ORCID: https://orcid.org/0000-0001-9599-5423

Universidade Federal Rural do Rio de Janeiro E-mail: willianbonnerj@hotmail.com

Fernanda De Jorge Gouvêa

ORCID: https://orcid.org/0000-0001-6102-926X

Universidade Federal Rural do Rio de Janeiro E-mail: fernandadjgouvea@gmail.com

Rodrigo Vasconcelos de Oliveira

ORCID: https://orcid.org/0000-0002-7420-1097

Universidade Federal Rural do Rio de Janeiro, Brasil.

E-mail: oliveirarvetal@gmail.com

\section{Resumo}

A agropecuária familiar promove a permanência do homem no campo, eleva a oferta de alimentos frescos e de qualidade para a sociedade. A extensão universitária destaca-se por fortalecer este nicho, devido a socialização do conhecimento e democratização dos espaços acadêmicos, com aceitação de paradigmas e concepções sociais, ambientais, econômicos e culturais. Atividades de extensão rural universitária favorecem a implementação de práticas agropecuárias sustentáveis, o uso dos recursos naturais disponíveis, a valorização do trabalho rural, e a desmarginalização da agricultura familiar. A extensão universitária associada às ciências agrárias, alia ensino, pesquisa 
e extensão rural, pela interação das instituições de ensino com a comunidade. No presente estudo foram realizados dois dias de campo no Setor de Caprinocultura da Universidade Federal Rural do Rio de Janeiro (UFRRJ), com identificação do perfil de 80 participantes, pela avaliação de fichas de inscrição e questionários. O público-alvo foi constituído por discentes em ciências agrárias, profissionais técnicos de ciências agrárias e agricultores familiares interessados em conhecimento e atualização em boas práticas na produção de cabras leiteiras. A identificação dos parâmetros de sustentabilidade nas propriedades rurais sinalizou a importância de apoio nas etapas de adequação higiênico-sanitárias dos produtos e comercialização, de modo a evitar a clandestinidade e riscos alimentares. As atividades de extensão universitária direcionadas às ciências agrárias proporcionaram a interação entre a comunidade acadêmica e a sociedade, o que promove uma formação acadêmica de qualidade, com benefícios associados às boas práticas e sustentabilidade do sistema de produção.

Palavras-chave: Agricultores familiares; Capacitação; Extensão rural.

\begin{abstract}
Family farming promotes the permanence of man in the countryside, and increases the supply of fresh and quality food. University extension activities enable social, environmental, economic, and cultural advances, a consequence of the socialization of knowledge, democratization of academic spaces, and acceptance of new paradigms. These favor the implementation of sustainable agricultural practices and the use of available natural resources, in addition to reducing the devaluation of rural work and the marginalization of family farming. The university extension associated with agricultural sciences in the theme of Good Practices in the Production of dairy goats, combines teaching, research and rural extension, through the interaction of educational institutions and the community. The holding of events in the Goat Farming Sector of the Federal Rural University of Rio de Janeiro (UFRRJ), promoted the flow of knowledge among farmers, technicians or students, with the improvement of animal production. There were 80 enrollments, mostly young people, students in agrarian sciences, followed by technical professionals from agrarian and family farmers, interested in knowledge and staying up to date, which helps choosing future strategies to facilitate learning. The identification of sustainability parameters in agricultural properties signaled the importance of supporting in the stages of adaptation and marketing, in order to avoid illegality and food risks. University extension activities aimed at agricultural sciences and dairy goat farming, provide quality professional training, in addition to benefits in the routine of family farmers, in which Good Practices improve the quality of milk and the sustainability of the production system.
\end{abstract}

Keywords: Familiar smallholders; Instruction; Rural extension.

\title{
Resumen
}

La agricultura familiar promueve la permanencia del hombre en el campo y aumenta la oferta de alimentos frescos y de calidad. En este contexto, las actividades que tienen objetivo de promover la interacción entre la comunidad académica y los agricultores familiares, posibilitan avances sociales, ambientales, económicos y culturales, consecuencia de la socialización del conocimiento, la democratización de los espacios académicos y la aceptación de nuevos paradigmas. Estos favorecen la implementación de prácticas agrícolas sostenibles y el uso de los recursos naturales disponibles, además de reducir la devaluación del trabajo rural y la marginación de la agricultura familiar. La incorporación universitaria, asociada a las ciencias agrarias en el tema de Buenas Prácticas en la Producción de cabras lecheras, combina docencia, investigación y extensión rural, a través de la interacción de las instituciones educativas y la comunidad. La realización de eventos en el Sector Caprino de la Universidad Federal Rural de Río de Janeiro (UFRRJ), promovió el flujo de conocimiento entre agricultores, técnicos o estudiantes, con el mejoramiento de la producción animal. Hubo 80 inscripciones, en su mayoría jóvenes, estudiantes de ciencias agrarias, seguidos de profesionales técnicos de la agricultura agraria y familiar, interesados en el conocimiento y la actualización, lo que ayuda a elegir estrategias futuras para facilitar el aprendizaje. La identificación de parámetros de sostenibilidad en predios agrícolas señaló la importancia de apoyar a los agricultores en las etapas de adaptación y comercialización de sus productos, a fin de evitar la ilegalidad y los riesgos alimentarios. Las actividades de extensión universitaria orientadas a las ciencias agrarias y la ganadería caprina lechera, brindan una formación profesional de calidad, además de beneficios en la rutina de los agricultores familiares, en los que las Buenas Prácticas mejoran la calidad de la leche y la sostenibilidad del sistema productivo.

Palabras clave: Agricultores familiares; Formación; Extensión rural.

\section{Introdução}

Ações universitárias integram ensino, pesquisa e extensão, para que a formação profissional esteja em consonância com as demandas da sociedade (Almeida, Peres \& Figueiredo, 2016). O Plano Nacional de Extensão possibilitou a institucionalização e ampliação do orçamento público destinado às ações de extensão nas instituições de ensino superior brasileiras (Rodrigues, 2015), estratégico para a formação de extensionistas na área de ciências agrárias, por meio da interação com profissionais de formação específica, para promoção de uma assistência técnica rural interdisciplinar (Landini, 2015). 
A formação de profissionais ligados às ciências agrárias deve capacitar para o desenvolvimento em sistemas de produção em bases sustentáveis, pela redução de insumos, e oferta de alimentos seguros. Segundo Rezaei-Moghaddam, Vatankhah \& Ajili (2020) a adesão ao uso de tecnologias limpas e pró-ambientais são instrumentos cruciais para a produção de alimentos saudáveis. Wang et al. (2020) afirmaram que práticas agropecuárias adequadas proporcionam eficiência, com redução dos impactos ambientais decorrentes da adoção de insumos químicos sintéticos, favorável à sustentabilidade de sistemas de produção.

Para um melhor aproveitamento das potencialidades de produção de alimentos é fundamental aliar o conhecimento técnico e científico ao conhecimento dos agricultores, em benefício a população local, social e ambientalmente, em respeito à diversidade da cultura local e dos recursos naturais. Assim, espaços de convivência entre agricultores e as instituições de pesquisa viabilizam o desenvolvimento tecnológico de forma dialógica e participativa (Oliveira, Wehrmann \& Sauer, 2015). De acordo com Souza, de et al. (2014), ferramentas metodológicas participativas de sustentabilidade, em pesquisa-ação com base qualitativa, quando incorporadas às atividades extensionistas de boas práticas de produção, possibilitam uma alteração positiva da percepção e do comportamento dos agricultores familiares, tornando-os autônomos e independentes.

A saber, os parâmetros de sustentabilidade são cerceados pelas condições ecológicas, socioeconômicas e culturais, com base na construção de agriculturas que incorporem princípios agroecológicos e na participação significativa de pequenos agricultores e de seus familiares (Abreu et al., 2012). A classe camponesa no Brasil tem sua história marcada pela exclusão, desapropriação e expropriação em consequência de políticas agrícolas favoráveis principalmente ao latifúndio e aos agronegócios de grande porte. A designação "agricultores familiares" foi incorporada pela legislação para organizar a acessibilidade a programas de fomento. Ressalva ao dever do Estado, ações de políticas inclusivas na mediação e implementação de ações em atendimento aos movimentos sociais brasileiros, ligados a lutas pela terra e reforma agrária (Pobreza E, 2013).

A criação de cabras para produção de leite é uma atividade agropecuária desenvolvida em diversos países do mundo (Pulina et al., 2018). O Brasil possui um rebanho caprino com aproximadamente 11 milhões de animais (IBGE, 2019) apresentando o maior rebanho da América do Sul (FAOSTAT, 2016). Nacionalmente, embora mais de 90\% dos caprinos estejam sendo criados na região Nordeste, a região Sudeste possui destaque na Caprinocultura Leiteira Intensiva. Especificamente, o Rio de Janeiro possui em torno de 13.600 cabeças de caprinos (IBGE, 2019).

Observa-se que para o incremento de lucratividade de pequenos e médios caprinocultores leiteiros, associações, cooperativas, as ações de extensão rural e atividades de pesquisa regionais são aspectos relevantes e necessários (Silva et al., 2013; Riet-Correia et al., 2013). Assim, o desenvolvimento da cadeia produtiva do leite caprino demanda amplas ações governamentais e privadas relacionadas aos agentes: estabelecimentos de insumos as propriedades rurais, produtores, estabelecimentos de venda de leite de cabra e seus derivados e até atravessadores (Heidorn et al., 2020).

A produção e consumo de alimentos reflete na qualidade de vida; e a oferta de alimentos saudáveis e seguros aos consumidores, exige aos técnicos e agricultores familiares, a implemententação de boas práticas de produção. Neste contexto, a agricultura familiar voltada para produção de alimentos de origem vegetal e animal tem se destacado como uma fonte importante de geração de renda, segurança alimentar e fortalecimento de práticas agroecológicas. Considerando o exposto, é necessária a promoção de espaços de convivência, e troca de conhecimentos, entre agricultores familiares, futuros profissionais das ciências agrárias e agentes de assistência técnica, por meio de atividades como os Dias de Campo. Estes possibilitam a interação e sensibilização dos participantes, pela aquisição de experiências, afinidades, e encurtamento dos espaços entre os atores das atividades extensionistas.

O Estado do Rio de Janeiro possui uma expressiva atuação de agricultores familiares, porém apresenta uma cadeia produtiva do leite de cabra que ainda carece de maior organização entre seus agentes. Desta forma, o presente estudo analisou 
dois eventos presenciais realizados no Setor de Caprinocultura da Universidade Federal Rural do Rio de Janeiro (UFRRJ) e o perfil do público alvo participante, em relação a sua faixa etária, gênero, interesse na atividade, suas ocupações e expectativas, e identificação dos parâmetros de sustentabilidade dos agricultores familiares assistidos, com o objetivo de identificar a troca cooperativa de conhecimento entre a Universidade e os agentes da cadeia produtiva do leite de cabra, e ações de extensão adequadas as demandas da Caprinocultura Leiteira, pelo intercâmbio de informações e/ou tecnologias entre os agentes envolvidos, visando autonomia, competência e ética.

\section{Metodologia}

As Boas Práticas no manejo de cabras leiteiras foram abordadas em dois Dias de Campo realizados no Setor de Caprinocultura da Universidade Federal Rural do Rio de Janeiro, parte do cronograma de execução de atividades de Projetos de Extensão (BIEXT) aprovados na referida instituição. Oficinas práticas na área de Ciências Agrárias, do campo à mesa, conforme ilustrado na Tabela 1, contemplaram a participação de agricultores familiares, discentes da UFRRJ e do Colégio Técnico da Universidade Rural (CTUR), profissionais das ciências agrárias, a comunidade acadêmica, município de Seropédica e adjacências, e a UFRRJ.

Tabela 1: Plano de atividade realizadas nos Dias de Campo I e II

\begin{tabular}{|c|c|c|}
\hline Atividades & I Dia de Campo & II Dia de campo \\
\hline & Sanidade; & Nutrição e alimentação de cabras leiteiras; \\
\hline Palestras (docentes) & Manejo nutricional e ambiência. & $\begin{array}{l}\text { Conservação de forragens (ensilagem de } \\
\text { cobertura); }\end{array}$ \\
\hline \multirow{9}{*}{ Oficinas (discentes) } & & Manejo reprodutivo e sanitário. \\
\hline & \multirow{8}{*}{$\begin{array}{l}\text { Características do leite de cabra; Degustação de } \\
\text { queijos, iogurtes, canjica doce, mingau doce, arroz } \\
\text { doce e bolos; } \\
\text { Obtenção higiênica do leite; Conservação de } \\
\text { forragens, com visitação a unidade demonstrativa } \\
\text { de Forrageiras e Leguminosas da UFRRJ, Instituto } \\
\text { de Zootecnia; } \\
\text { Casqueamento de caprinos. }\end{array}$} & $\begin{array}{l}\text { Fabricação do leite condensado e brigadeiro; } \\
\text { Fabricação de licor de laranja e trufas com leite de } \\
\text { cabra; }\end{array}$ \\
\hline & & Casqueamento de cabras; \\
\hline & & Fabricação de doce de leite e iogurte; \\
\hline & & Famacha; \\
\hline & & Avaliação de condição corporal dentaria; \\
\hline & & Fabricação de doce de leite; \\
\hline & & Obtenção higiênica de leite; \\
\hline & & Cashmere de cabra; \\
\hline
\end{tabular}

Fonte: Autores.

Meios de divulgação existentes na UFRRJ, Secretaria de Agricultura, Associação de Moradores do Município e Emater, com elaboração de cartaz para divulgação física foram utilizados para o convite ao público alvo, através de preenchimento de ficha de inscrição padronizada para identificação do perfil, por meio de informações sobre idade, profissão, meios de divulgação, município, interesse e expectativas de participação.

As atividades iniciaram pela manhã, com formação em roda, para apresentação dos presentes. As oficinas foram distribuídas no período da manhã e da tarde. Os participantes receberam alimentação (café, almoço e lanche), degustação e amostras dos produtos das oficinas de beneficiamento do leite de cabras, mudas por estaquias de gliricídeas, e sementes de leguminosas de feijão guandú e macrotiloma.

Dentre os participantes, oito agricultores familiares foram entrevistados para avaliação dos indicadores de sustentabilidade, por meio de formulário, proposto por Sá et al. (2012), em quatro dimensões de análises: a social, a econômica, a ambiental e a política. A escala de variação foi dada pelo parâmetro adotado, entre mais ou menos sustentável. Na dimensão social, os indicadores observados foram: moradia por condições de saneamento e luz; anos de estudo formal; e responsabilidade intergeracional. Na dimensão ambiental foram analisados como indicadores de sustentabilidade: a 
contaminação ambiental; desmatamento; e diversidade dos sistemas de produção. Para a avaliação da dimensão política, avaliou-se a participação popular dos agricultores familiares, pela identificação do tipo de associativismo; seguido da acessibilidade à assistência técnica; e financiamento. Na dimensão econômica, o grau de legalidade x clandestinidade, e a infraestrutura foram avaliados, com efeito na sustentabilidade dos sistemas de produção.

\section{Resultados e Discussão}

Nos Dias de Campo de Boas Práticas no Manejo de Cabras Leiteiras associada as ciências agrárias realizados na Universidade Federal Rural do Rio de Janeiro, foram registradas 80 inscrições, correspondentes a 30 no I Dia de Campo (IDC) e 50 inscrições no II no Dia de Campo (IIDC). Nas Figuras 1 e 2 estão ilustradas as oficinas e a $1^{\text {a }}$ Palestra sobre manejos nutricionais e principais forrageiras utilizadas na alimentação de caprinos. A Figura 3 ilustra as oficinas e a $2^{\circ}$ Palestra sobre manejo reprodutivo. A Figura 4 ilustra a apresentação dos produtos lácteos de cabras.

Figura 1-Características do leite de cabra e produtos (esquerda); boas práticas aplicadas na ordenha (direita).

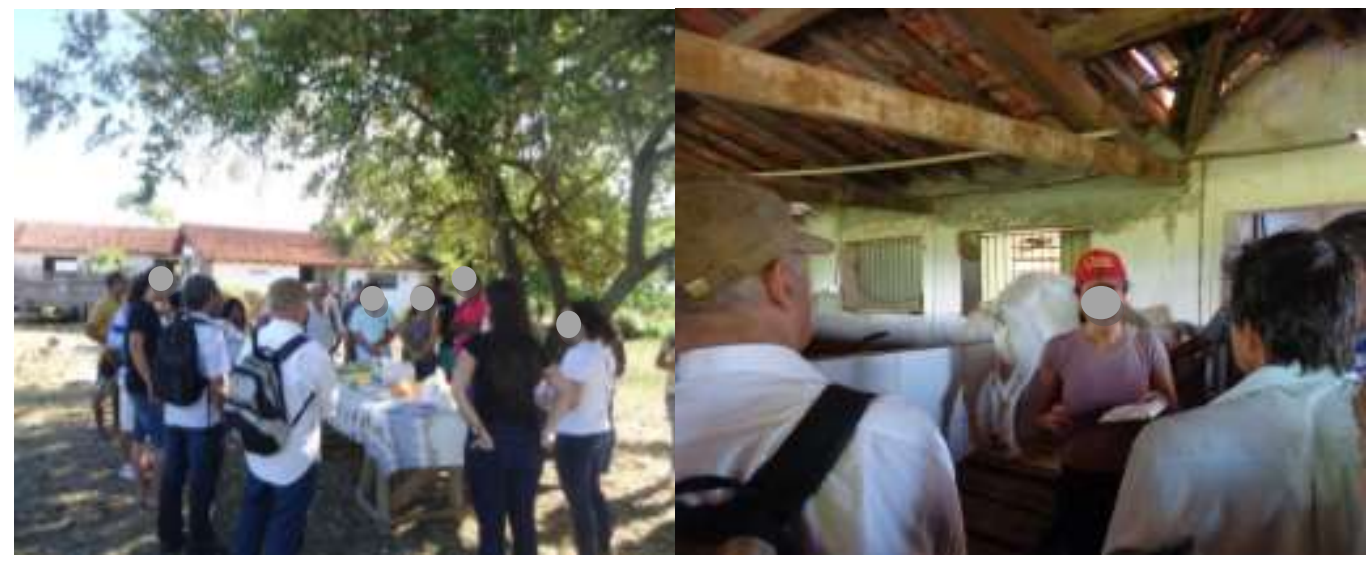

Fonte: Autores.

Figura 2- Manejo nutricional e forrageiras utilizadas na alimentação de caprinos.

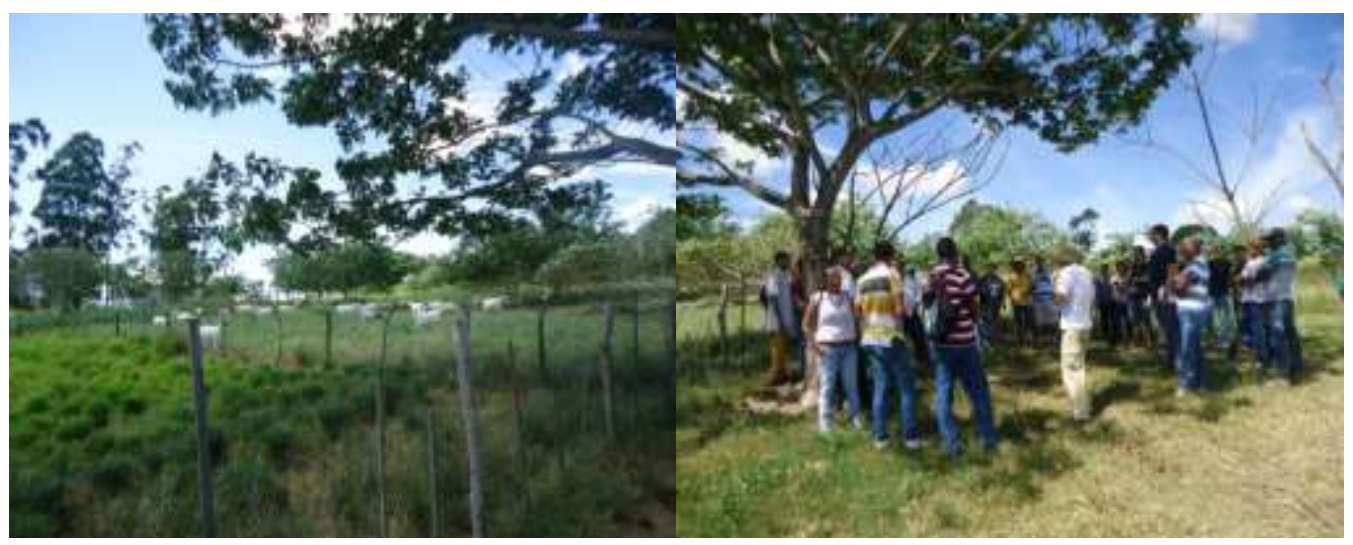

Fonte: Autores. 
Figura 3-Campo experimental de plantas forrageiras (esquerda); manejo reprodutivo e sanitário (direita).

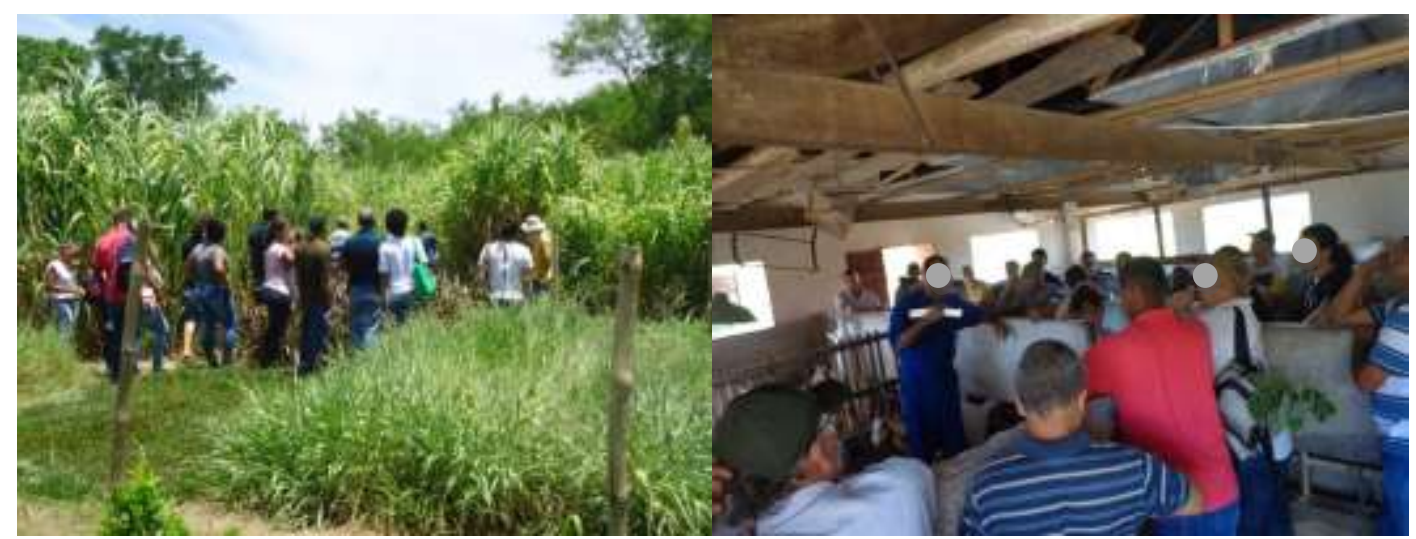

Fonte: Autores.

Figura 4 -Oficina de lácteos de cabras.

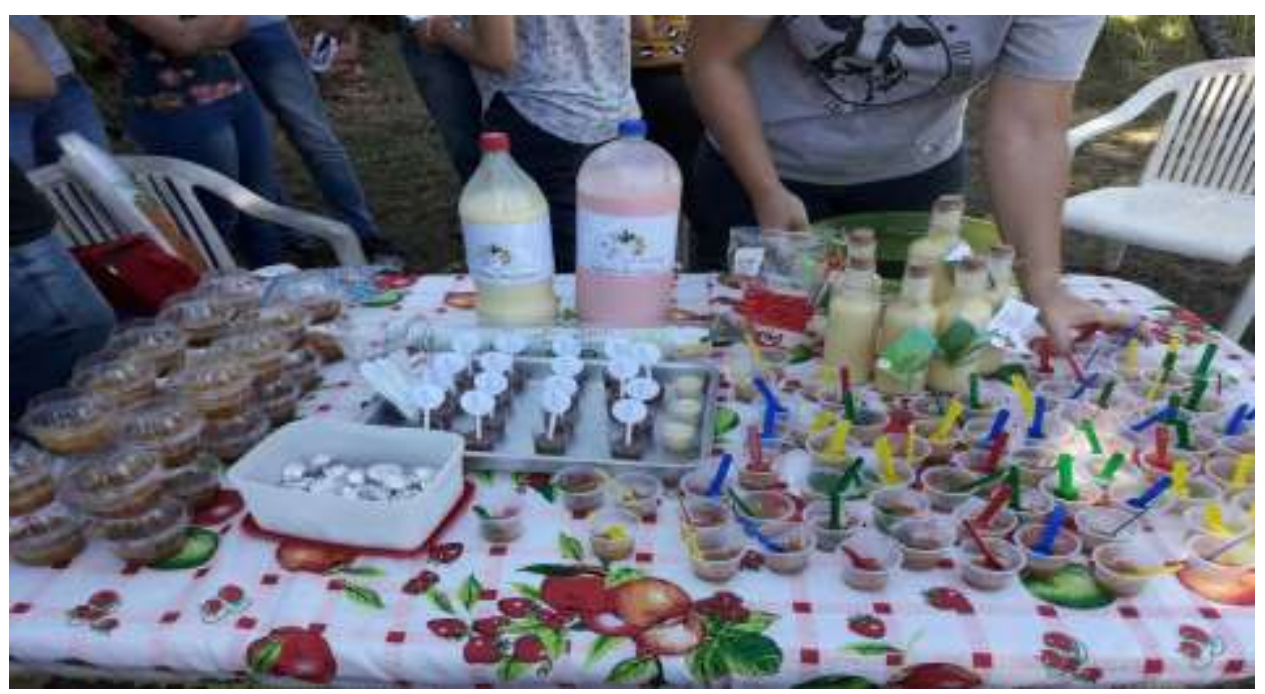

Fonte: Autores.

As fichas de inscrição padronizadas constaram de informações sobre idade, profissão, meios de divulgação, município, interesse e expectativas do participante, cujas informações foram ordenadas nas figuras 5-10, a seguir.

O perfil etário (Figura 5) apresentou variações entre os eventos. No IDC prevaleceu o gênero masculino (70\%), em detrimento do feminino (30\%), porém no IIDC, a participação do gênero masculino (40\%) foi inferior ao gênero feminino (60\%), mais notória na faixa etária entre 16 e 26 anos (18\% masculino x 46\% feminino), o que reflete o interesse de discentes do gênero feminino nos cursos de ciências agrárias, da referida instituição. 
Figura 5: Perfil do público-alvo quanto ao gênero e faixa etária.

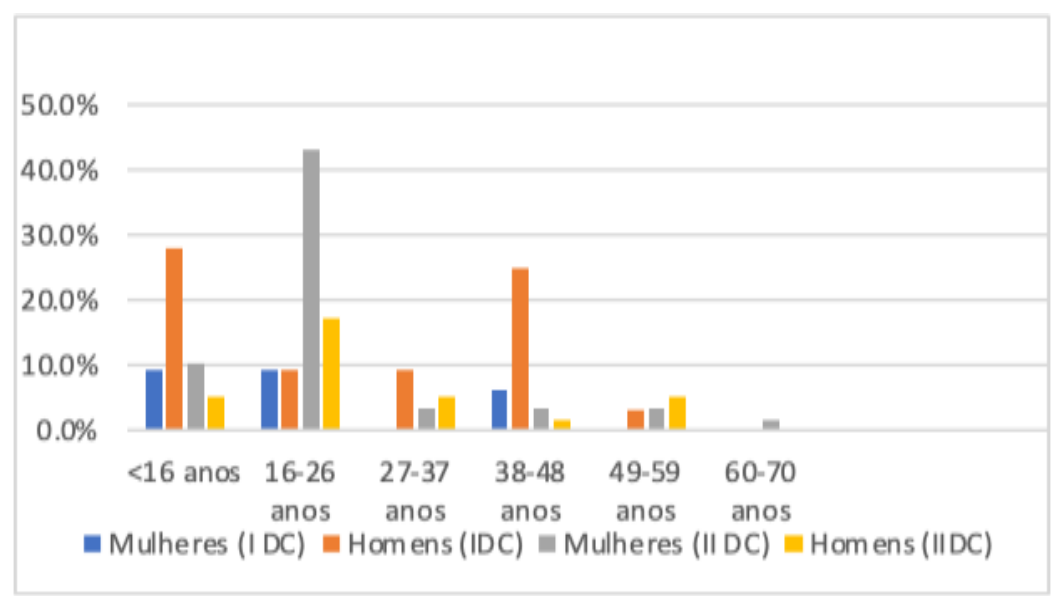

Fonte: Autores.

A presença de participantes nas faixas etárias inferiores a 16 anos sinaliza para o público jovem, o que é relevante, visto que com a metropolização da baixada fluminense, jovens são estimulados a ter ofícios desvinculados a agricultura. Assim, a valorização da atividade no campo favorece a manutenção desses jovens na atividade rural, em consonância a aptidão de seus familiares, visto o histórico de uso da terra. O que motiva a ações de extensão para a comunidade local, afim de democratizar o espaço acadêmico, e estimular o ingresso de graduandos oriundos dos municípios adjacentes à instituição, promissor para o desenvolvimento social, econômico e ambiental da região.

Leila \& Fernandes (2014) ressaltaram a importância de as mulheres conhecerem as Boas Práticas para uma alimentação de qualidade; decorrente de sua função social de educar os filhos, inclusive no que tange à alimentação, com valorização da variedade e do potencial nutricional. As pesquisadoras apontam a falta de técnicas e conhecimento adequados às agricultoras familiares, com interferência na produção e práticas alimentares do cotidiano, relacionadas a oferta e aceitação de alimentos.

As desigualdades nas relações de gênero na agricultura familiar constituem um obstáculo ético e prático ao desenvolvimento rural, manifestando-se na distribuição das riquezas geradas pelo trabalho familiar, e na participação em processos decisórios familiares. A oferta de espaços de sociabilidade, o acesso à informação, e aos bens culturais, são formas de empoderar as mulheres por meio de dinâmicas sociais, através da aquisição de técnicas adequadas aos sistemas de produção, e implicação direta nas práticas alimentares de seus familiares.

Estes dados permitem um melhor direcionamento das tecnologias sociais que possam ser aplicadas para o fortalecimento social deste público, com observância da regularidade de participação nos eventos, e adequação às expectativas, e ajustes à futuras atividades.

Dentre os meios de divulgação (Figura 6), as conversas presenciais ou via redes sociais, principalmente via aplicativo WhatsApp representaram 39\% das inscrições, seguido da produção do material gráfico (cartaz), equivalente à 37,5\% do total de inscrições: $17,5 \%$ no IDC e $20 \%$ no IIDC. 
Figura 6: Efetividade dos Canais de Comunicação.

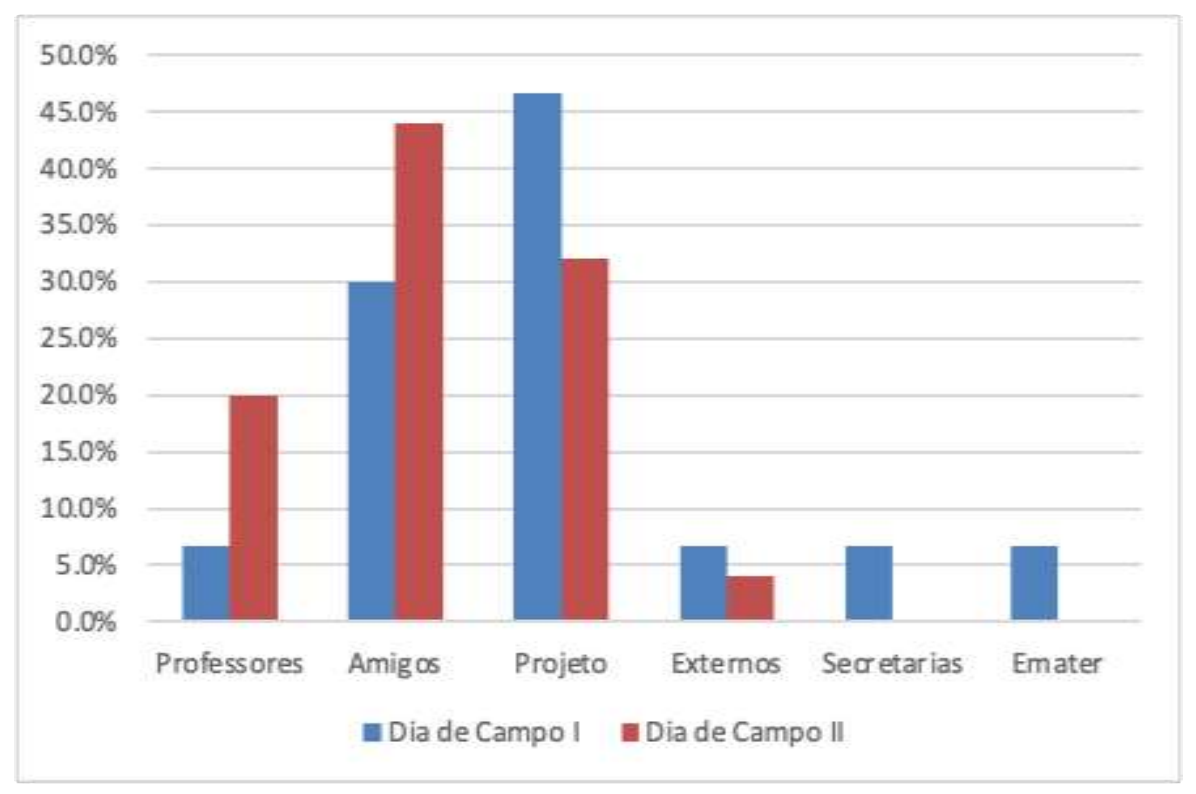

Fonte: Autores.

Em relação ao alcance regional (Figura 7) 16 diferentes regiões foram observadas: Seropédica, Paulo de Frontin, Paracambi, Magé, Mangaratiba, Japeri, Angra dos Reis, Itacuruçá, Cachoeiro de Itapemirim, Itaguaí, Belford Roxo, Nova Friburgo, e no município do Rio de Janeiro, Vaz Lobo, Jacarepaguá, Guadalupe, e residentes no alojamento universitário oriundos de outros estados como, São Paulo, Minas Gerais e Fortaleza. As diferentes localidades pleiteadas pelo evento permitiram um maior alcance das atividades ligadas a cadeia produtiva de cabras leiteiras, a fim de elevar a quantidade $\mathrm{e}$ qualidade dos derivados lácteos, concomitante à conscientização da importância das Boas Práticas na Produção Animal.

Ressalta-se que a criação de cabras leiteiras é uma atividade rentável capaz de ser desenvolvida em propriedades rurais de menor área quando comparada as utilizadas para bovinocultura leiteira (Ribeiro, 1997). Adicionalmente, o leite de cabra é um produto de elevado valor nutritivo rico em aminoácidos essenciais e de alta digestibilidade sendo interessante para criança e idosos (Cenachi et al., 2011). O leite caprino ainda possui um baixo alergênico sendo uma opção para pessoas alérgicas ao leite de vaca. Entretanto, no estado do Rio de Janeiro existe uma carência atual de organização dos caprinocultores leiteiros e dos demais agentes da cadeia de produção do leite de cabra e seus derivados.

Seropédica possui características agrárias, com predominância de unidades de base familiar, relevante para a economia do município, e fonte de renda para a agricultura familiar; ponto chave para o desenvolvimento rural sustentável, como fortalecimento econômico e empoderamento das famílias (De, Frota R. C., 2016). Os aspectos sociais, ambientais, e a garantia de alimentos saudáveis para a esta região, continuam expressivos e carecem da atenção, não só do poder público como também das instituições de pesquisa que nela se situam (Fagundes, 2017).

A agricultura familiar, composta por pequenos e médios produtores rurais, responde pela maior parte da produção de alimentos básicos no Brasil, responsáveis pelo abastecimento urbano, com diversificação de suas atividades e beneficiamento de matérias-primas (Completo et al., 2013). E os consumidores reconhecem a qualidade e a promoção de saúde dos produtos, o não uso de agrotóxicos, a certificação, e a acessibilidade (Souza, Melo \& Silva, 2018). 
Figura 7: Alcance regional.

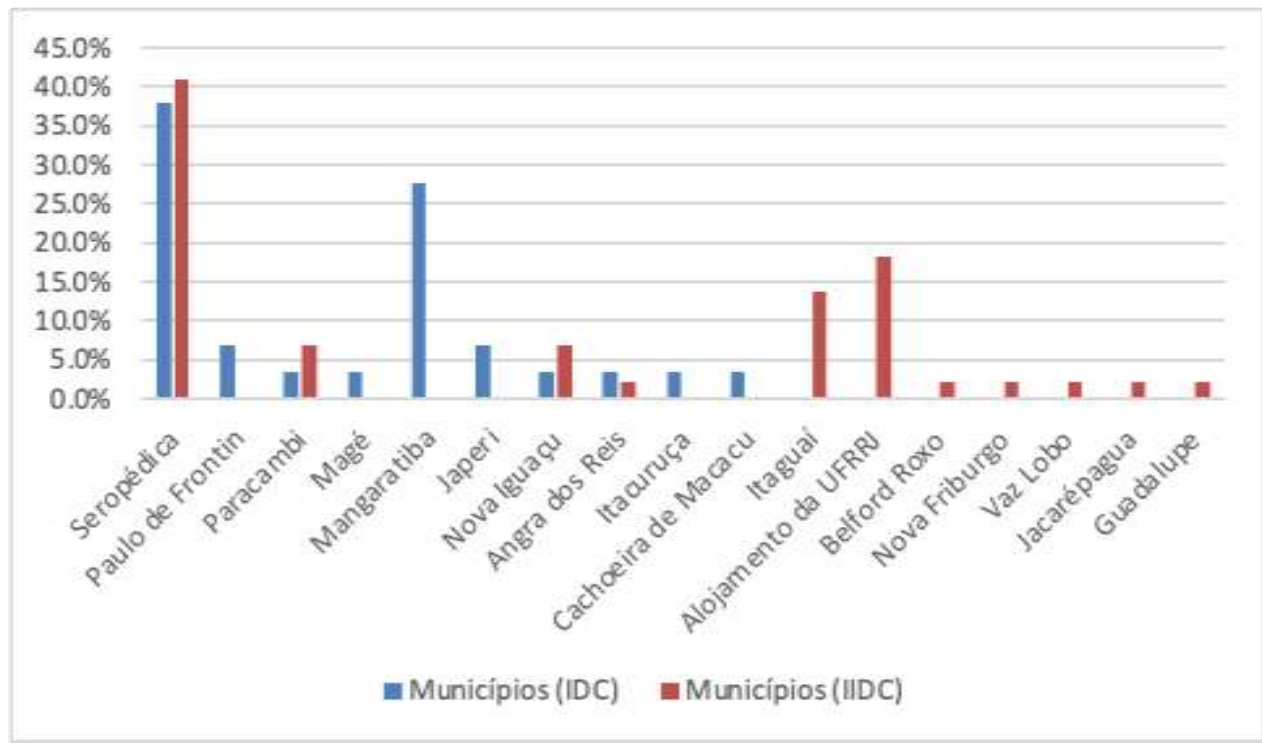

Fonte: Autores.

Assim, observou-se que o desenvolvimento de práticas de extensão universitária associado às ciências agrárias viabilizam a qualificação profissional dos discentes, e ampliam o alcance dos benefícios relacionados às Boas Práticas de Produção, com abordagem de estratégias desde o melhoramento zootécnico do rebanho, ao incremento da qualidade do produto principal, o leite.

Dentre as ocupações dos participantes (Figura 8), 20 diferentes categorias foram identificadas. Esta multiplicidade de ofícios expressa a diversidade do público interessado nas atividades de extensão voltadas a caprinocultura leiteira, e a abrangência dos eventos. Discentes de graduação corresponderam a 50\% dos participantes, seguidos de profissionais técnicos das agrárias (10\%) e agricultores familiares (7,5\%), o que permite a interação dos principais agentes envolvidos na extensão universitária nas ciências agrárias. Apesar de pouco expressiva a participação de agriculotes familiares, nota-se dentre os participantes, criadores cuja atividade principal é outra, senão a agropecuária, tendo esta como expectativa de crescimento.

A interação entre agricultores familiares, pequenos produtores rurais, técnicos agropecuários, e acadêmicos em cursos de ciências agrárias, tornaram a atividade interativa e produtiva. Os discentes ministraram oficinas temáticas, comprometidos desde a obtenção e preparo dos produtos lácteos; apresentação oral; produção de material teórico; e entrega de folders; oportunidade para treinar a confiança, empatia e aprimoramento técnico. No I Dia de Campo, quatro oficinas foram ministradas por quatro discentes do curso de zootecnia. No II Dia de Campo nove oficinas foram ministradas em duplas, por discentes dos cursos de ciências agrárias pertencentes aos cursos de zootecnia, agronomia, medicina veterinária e ciências agrícolas. 
Figura 8: Perfil ocupacional dos participantes.

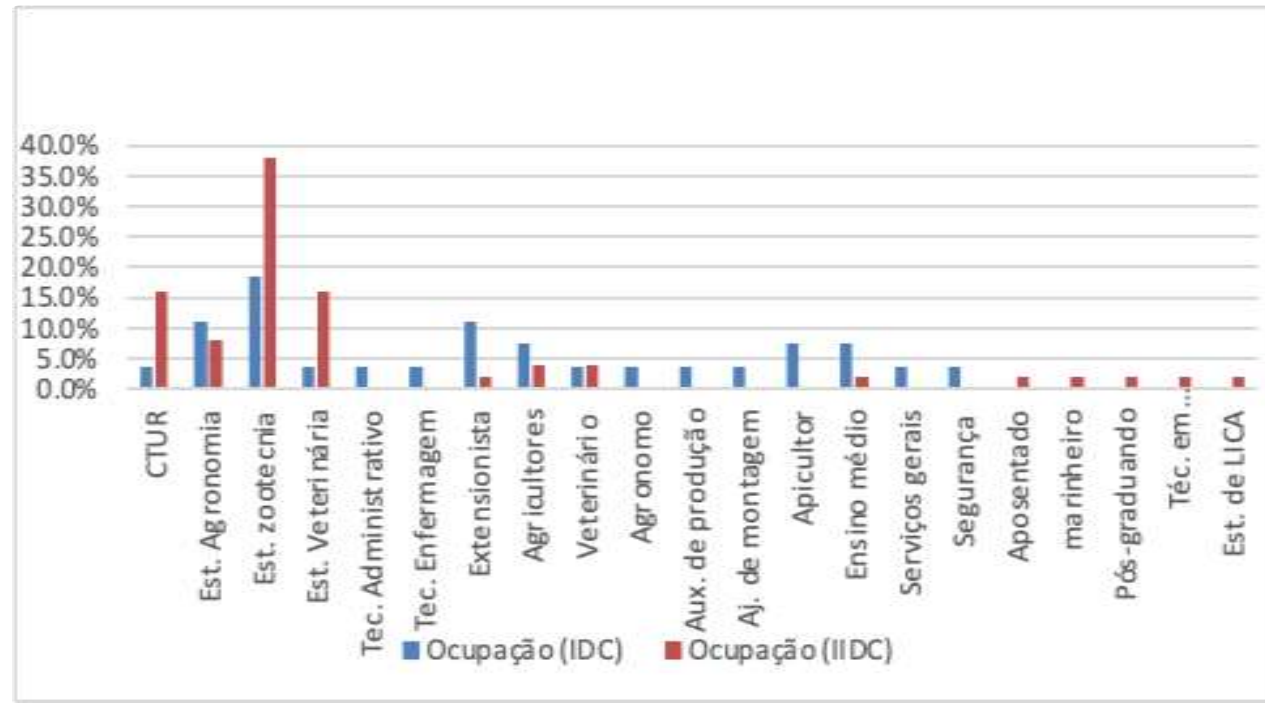

Fonte: Autores.

Os perfis de interesse (Figura 9) em participação no evento foram identificados, e auxiliam na tomada de decisões em futuras abordagens. As técnicas utilizadas na agropecuária familiar, são normalmente adquiridas informalmente ou por meio de atividades de extensão, como dias de campo ou visitas técnicas. A demanda por conhecimento do público-alvo apontou a necessidade de constante oferta de treinamentos e capacitações, para o desenvolvimento sustentável dos sistemas produtivos.

Os agricultores familiares são os alvos, e ao mesmo tempo agentes de extensão, visto que sinalizam os principais entraves na prática das atividades, e ao mesmo tempo auxiliam no apontamento de soluções simples e viáveis, que visem a promoção de alimentos seguros e sistemas com bases agroecológicas. Almeida, Peres \& Figueiredo (2016) elucidaram a importância das ações de extensão universitária para a formação de profissionais de áreas agrárias, cuja vivência com agricultores familiares resulta em intenso aprendizado, e em outro olhar para as questões da agricultura familiar, com abertura para o entendimento das dificuldades e limitações, conservação ambiental, responsabilidade social e retorno econômico.

Figura 9: Perfil do interesse no evento dos participantes.

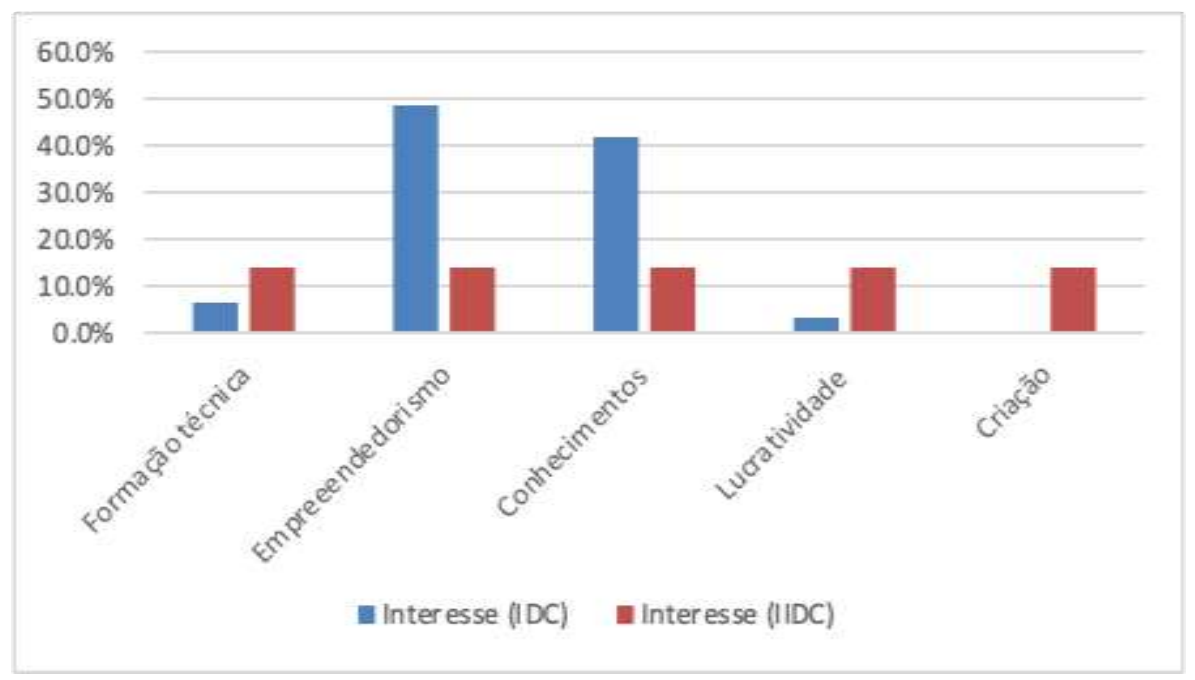

Fonte: Autores. 
A busca por conhecimentos práticos e inovações sustentáveis representam as pricipais expectativas (Figura 10) em Boas pràticas de Cabras Leiteiras. A promoção da capacitação dos técnicos com atividades de extensão vinculadas às ciências agrárias, assim como o apoio aos agricultores e produtores rurais, são práticas que fortalecem os elos do sistema produtivo, e aprimoram as ações e resultados esperados, pelo estabelecimento de parâmetros e metas pertinentes.

Figura 10: Expectativa do público-alvo nas Boas Práticas de Cabras leiteiras.

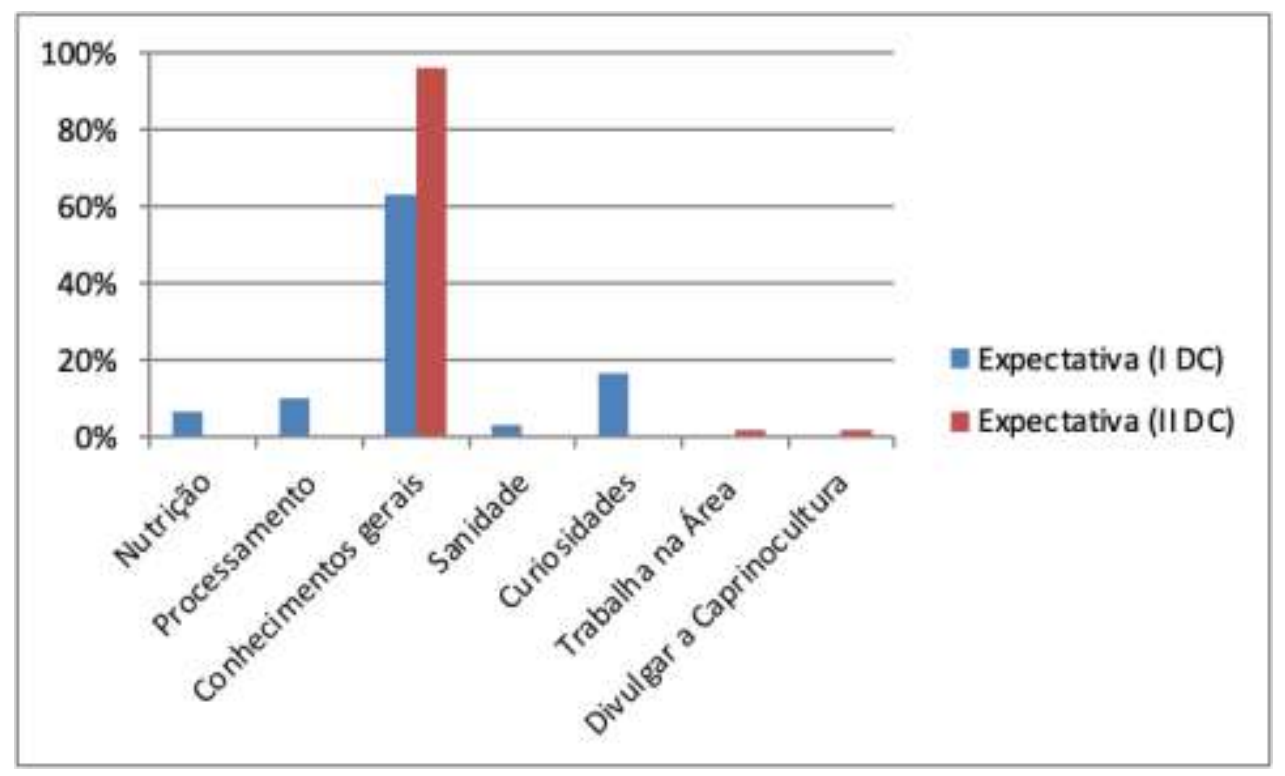

Fonte: Autores.

Martins et al. (2015) realizaram diagnóstico de Boas Práticas de Produção de agricultores familiares, e identificaram a ausência de treinamentos em Boas Práticas de Produção, o que corroborou para que nenhuma das propriedades atendidas cumprisse o total das exigências do Protocolo de Boas Práticas de Produção, com implicações nas questões administrativas, na qualidade dos alimentos, e na proteção ambiental do sistema produtivo.

Constata-se, entre os agricultores, uma preocupação permanente em garantir a reprodução da unidade familiar, com a presença de valores socioeconômicos, de cunho cultural e ambiental (Lima \& Vargas, 2015). Kerber \& Neto (2007) destacam que os agricultores familiares não são avessos às inovações, mas sim, agentes inovadores, experimentador constante, para o desenvolvimento de novas rotinas das atividades agrícolas, incorporadas ao sistema de produção, de forma comunitária.

As práticas sociais devem ser baseadas na perpectiva do público alvo, portanto mais efetivas, e replicáveis a outras realidades (Sousa et al., 2019). Tecnologias Sociais promovem o desenvolvimento sustentável, concomitante a promoção da saúde e redução da pobreza, em auxilío à manutenção dos saberes, e aquisição de novos conhecimentos (E. F. Oliveira et al., 2015).

Dentre os parâmetros de sutentabilidade avaliados, $41 \%$ dos resultados foram ótimos para a dimensão social, principalmente com relação a escolaridade acima de 10 anos; e 25\% foram insatisfatórios, relacionados à responsabilidade intergeracional, filhos de agricultores familiares que não pretendem dar continuidade às atividades no campo.

Na dimensão ambiental, 50\% dos resultados foram ótimos ou médio, decorrentes principalmente da diversidade de culturas cultivadas, destaque para a observação da ocorrência de manejo pela queima. Na dimensão política $75 \%$ dos resultados foram insatisfatórios, devido a reduzida ocorrência de associativismo e assistência técnica, apesar de ocorrência de participação na Associação de Agricultores Biológicos do Rio de Janeiro (ABIO) e assistência técnica rural pela Emater.

Na dimensão econômica, $100 \%$ dos resultados foram ruins, o que compromete a legalidade dos produtos de origem 
animal, e relato de propriedade com a infraestrutura adequada à legislação vigente, na espera de liberação para comercialização de lácteos de cabra, com prática clandestina em feiras livres.

Nas dimensões política e econômica, os parâmetros de sustentabilidade foram mais críticos, e sinalizaram a importante participação e assistência, dos órgãos de fomento. Destaque para as adequações higiênico-sanitárias, e subsequente legislação dos produtos, temas a serem abordados em futuras ações de extensão, direcionadas a esse público. As Boas Práticas Agropecuárias elevam a sustentabilidade dos sistemas produtivos, associado à valorização da atividade rural.

Além de fatores sociais, econômicos e institucionais, as políticas que visam promover a produção agropecuária sustentável devem abordar as dimensões psicológicas da adoção da tecnologia, com práticas sociais baseadas na perspectiva do público alvo, tornando-as mais efetivas, e replicáveis a outras realidades ( $\mathrm{Li}$ et al., 2020). A sustentabilidade de sistemas de produção de agricultores familiares requerem metas como: a redução do analfabetismo, a valorização do saber local, a preservação ambiental, o fortalecimento organizacional, a realização de ações institucionais participativas e condizentes com a realidade do território e a inserção dos produtos familiares no mercado legal (Sá et al., 2012).

\section{Considerações Finais}

As Boas Práticas de Produção de Cabras Leiteiras associadas a extensão universitária aprimoraram a qualidade profissional de discentes e técnicos de ciências agrárias, com aquisição de conhecimentos técnicos e interação com agricultores familiares, o que aproxima as realidades através das trocas de experiências, e amplia as afinidades e percepções; essencial para a formação de futuros profissionais atuantes na extensão rural que atendam às demandas sociais, econômicas, e ambientais, inerentes à atividade.

A acessibilidade ao espaço acadêmico, democratização do conhecimento, e o intercâmbio de experiências entre os diferentes atores da produção agropecuária, agricultores familiares, comunidade acadêmica (futuros profissionais atuantes nas ciências agrárias), e comunidade regional, fundamentam a elaboração de soluções relacionadas ao cotidiano, de forma criativa e adequada aos sistemas locais de produção. Em destaque à necessidade de apoio ao atendimento dos requisitos higiênicosanitárias e tecnológicos, para a comercialização de produtos de origem animal, o que auxilia para o desenvolvimento socioeconômico e a qualidade de vida, de forma sustentável.

\section{Referências}

Abreu, L. S., Bellon, S., Brandenburg, A., Ollivier, G., Lamine, C., Darolt, M. R., \& Aventurier, P. (2012). Relações entre agricultura orgânica e agroecologia: desafios atuais em torno dos princípios da agroecologia. Desenvolvimento e Meio Ambiente, 26, 143-160. https://doi.org/10.5380/dma.v26i0.26865

Almeida, D., Peres, R. B., \& Figueiredo, A. N. (2016). Rural environmental planning in a family farm: education, extension and sustainability. Ciência Rural, 46(11), 2070-2076. https://doi.org/10.1590/0103-8478cr20150926

Cenachi, D. B. Furtado, m. A. B. Bell, m. J. V. Pereira, m. S. Garrido, 1. A. Pinto, m. A. O. (2011). Aspectos composicionais, propriedades funcionais, nutricionais e sensoriais do leite de cabra: uma revisão. Revista Instituto laticínio Cândido Tostes, no 382 v. 66 p. $12-20$ juiz de fora/MG.

Completo, A., Greczyszn, F. R., Correia, S., \& Favarão, M. (2013). Socio-Economic Profile of the Farmers Dealers Microregion of Campo Mourao-Pr. Campo Digital: Rev. Ciências Exatas e da Terra e Ciências Agrárias Revista Campo Digital, 8(1), 10-17. http://revista.grupointegrado.br/revista/index.php/campodigital

Souza, A. P., Honorato, L. A., Gómez, C. U., Cardoso, C. S., \& Hötzel, M. J. (2014). Construção e uso de indicadores para avaliação do manejo da ordenha: Uma proposta metodológica participativa. Ciencia Rural, 44(5), 911-917. https://doi.org/10.1590/S0103-84782014000500024

De, T. (2016). As potencialidades da agricultura familiar em Seropédica / RJ a partir da sistematização de experiências Introdução O município de Seropédica. “Este município está localizado dentro dos limites de latitude Sul $22{ }^{\circ} 44$ ' 38 ” e $43^{\circ} 42$ ' 28 ” de longitud.

Fagundes, G. T. (2017). Um estudo sobre ruralidades em Seropédica.

Food and Agriculture Organization of the United Nations. FAOSTAT (2016). Statistical Database.

Heidorn, 1. 1., Souza, C. B.; Nascimento, A. R., Wander, A. E. (2020) Sustentabilidade das cadeias produtivas de leite de cabra e ovelha: uma revisão sistemática com base no Triple Bottom Line. In: Congresso da Sociedade Brasileira de Economia, Administração e Sociologia rural, 58., 2020, Foz do Iguaçu. 
Cooperativismo, inovação e sustentabilidade para o desenvolvimento rural: anais... Foz do Iguaçu: UNIOESTE.

IBGE - Instituto Brasileiro de Geografia. (2019) - In: IBGE. Sidra: sistema IBGE de recuperação automática. Disponível em: http://www.sidra.ibge.gov.br

Kerber, M., \& Neto, C. C. (2007). Agricultura familiar e desenvolvimento rural: o caso da comunidade Vargem do Brasil, São Fidélis/RJ. Revista Brasileira de Agroecologia, 2(2), 367-371.

Landini, F. P. (2015). Problems faced by Brazilian rural extensionists and their relationship with their conception of rural extension/Problemas enfrentados por extensionistas rurais Brasileiros e sua relacao com suas concepcoes de extensao rural.(sociologia rural)(texto en por. Ciencia Rural, 45 (2), 371.

Leila, I., \& Fernandes, C. (2014). Produção e consumo de alimentos em assentamentos rurais : hábitos e práticas orientados para o alcance da segurança alimentar. 30-40.

Li, J., Feng, S., Luo, T., \& Guan, Z. (2020). What drives the adoption of sustainable production technology? Evidence from the large scale farming sector in East China. Journal of Cleaner Production, 257. https://doi.org/10.1016/j.jclepro.2020.120611

Lima, F. A. X., \& Vargas, L. P. (2015). Alternativas socioeconômicas para os agricultores familiares: O papel de uma associação agroecológica. Revista Ceres, 62(2), 159-166. https://doi.org/10.1590/0034-737X201562020005

Martins, J., Melo, M. C., De Oliveira Do Nascimento, K., Lucena, J., Júnior, B., Saldanha, T., Martins, M. I., \& Barbosa, J. (2015). Diagnóstico e qualidade microbiológica de ovos caipiras produzidos por agricultores familiares* Diagnosis and microbiological quality of eggs produced by rednecks farmers. 22(1), 48-53. https://doi.org/10.4322/rbcv.2015.319

Oliveira, E. F., Jesus, V. S., Siqueira, S. M., Alves, T. eA., Santos, I. M., \& Camargo, C. L. (2015). Promoting health in vulnerable communities: social technologies for poverty reduction and sustainable development . Revista gaúcha de enfermagem / EENFUFRGS, 36, 200-206. https://doi.org/10.1590/19831447.2015.esp.56705

Oliveira, M. N. da S., Wehrmann, M. E. S. D. F., \& Sauer, S. (2015). Agricultura Familiar no Distrito Federal: a busca por uma produção sustentável. Sustentabilidade em Debate, 6(1), 53. https://doi.org/10.18472/sustdeb.v6n1.2015.11422

Pobreza, C. À., \& E, O. B. M. (2013). Programa do leite em cabaceiras/pb: contrapontos entre os discursos de combate à pobreza, o banco mundial e políticas para o campo (Milk Program in the Cabaceiras/PB: counterpoints between speeches of poverty, the world bank and policies for the field). Cadernos Do Logepa, 8(1-2), 54-78.

Pulina, G.; Milán, M. J.; Lavín, M. P.; Theodoridis, A. (2018) Invited review: Current production trends, farm structures, and economics of the dairy sheep and goat sectors. Journal of Dairy Science, 101, n. 8, p. 6715-6729, 2018/08/01.

Rezaei-Moghaddam, K., Vatankhah, N., \& Ajili, A. (2020). Adoption of pro-environmental behaviors among farmers: application of Value-Belief-Norm theory. Chemical and Biological Technologies in Agriculture, 7(1), 1-15. https://doi.org/10.1186/s40538-019-0174-z

Ribeiro, S. D. de A. (1997) Criação Racional de Caprinos. São Paulo: Nobel. 318 p. ISBN: 85-213-0972-4.

Riet-Correa, B. et. al. (2013). Sistemas produtivos de caprinocultura leiteira no semiárido paraibano: caracterização, principais limitantes e avaliação de estratégias de intervenção. Pesquisa Veterinária Brasileira, Santa Maria, v. 33, n. 3, p. 345-352.

Rodrigues, M. (2015). O fórum de pró-reitores de extensão e sua contribuição no debate sobre a extensão universitária. $391-409$.

SÁ, C. O., MARINHO, G. L. de O. C., SÁ, J. L., ; RONER, M. N. B., NASCIMENTO, I. R., \& SÁ, F. P. (2012). Sustentabilidade dos sistemas de produção dos agricultores familiares e produtores de queijo em Nossa Senhora da Glória, semiárido sergipano. Revista Brasileira de Agroecologia, 7(3), 26 -39.

Silva, E.M.N.; Souza, B.B.; Silva, G.A.; Azevedo, S.S.; Gomes, T.L.S. (2013). Caracterização dos sistemas produtivos de leite de cabra nos cariris paraibano. Revista Caatinga, 26(1).

Sousa, I. V. de, Brasil, C. C. P., Silva, R. M. da, Vasconcelos, D. P. E., Vasconcelos Filho, J. E. de, Finan, T. J., Bezerra, I. N., \& Pinheiro, C. P. O. (2019). Enfrentamento de problemas que impactam na saúde de uma comunidade socialmente vulnerável sob a ótica dos moradores. Ciencia \& saude coletiva, 24(5), 1647-1656. https://doi.org/10.1590/1413-81232018245.04392019

Souza, L. N. de, Melo, M. B., \& Silva, D. W. (2018). Caracterização do perfil dos consumidores da Feira de Produtos Orgânicos do município de Santarém, Pará. Cadernos de Agroecologia, 13(1), 7. http://cadernos.aba-agroecologia.org.br/index.php/cadernos/article/view/44

Wang, J., Zhang, L., He, X., Zhang, Y., Wan, Y., Duan, S., Xu, C., Mao, X., Chen, X., \& Shi, X. (2020). Environmental mitigation potential by improved nutrient managements in pear (Pyrus pyrifolia L.) orchards based on life cycle assessment: A case study in the North China Plain. Journal of Cleaner Production, 262, 121273. https://doi.org/10.1016/j.jclepro.2020.121273 